The Open Dentistry Journal
CrossMark
Content list available at: www.benthamopen.com/TODENTJ/
DOI: $10.2174 / 1874210616021001360$

RESEARCH ARTICLE

\title{
Cationic Lipid Content in Liposome-Encapsulated Nisin Improves Sustainable Bactericidal Activity against Streptococcus mutans
}

\author{
Kazuo Yamakami ${ }^{1, *}$, Hideaki Tsumori ${ }^{2}$, Yoshitaka Shimizu ${ }^{3}$, Yutaka Sakurai ${ }^{1}$, Kohei Nagatoshi ${ }^{4}$ and \\ Kenji Sonomoto 5 \\ ${ }^{I}$ Department of Preventive Medicine and Public Health, National Defense Medical College, Tokorozawa, Japan \\ ${ }^{2}$ Department of Chemistry, National Defense Medical College, Tokorozawa, Japan \\ ${ }^{3}$ Department of Applied Biochemistry, Tokai University, Hiratsuka, Japan \\ ${ }^{4}$ Eco Friendly Institute Ltd., Fukuoka, Japan \\ ${ }^{5}$ Department of Bioscience and Biotechnology, Faculty of Agriculture, Graduate School, Kyushu University, Fukuoka, \\ Japan
}

Received: December 31, 2014

Revised: May 13, 2016

Accepted: June 24, 2016

\begin{abstract}
An oral infectious disease, dental caries, is caused by the cariogenic streptococci Streptococcus mutans. The expected preventive efficiency for prophylactics against dental caries is not yet completely observed. Nisin, a bacteriocin, has been demonstrated to be microbicidal against $S$. mutans, and liposome-encapsulated nisin improves preventive features that may be exploited for human oral health. Here we examined the bactericidal effect of charged lipids on nisin-loaded liposomes against $S$. mutans and inhibitory efficiency for insoluble glucan synthesis by the streptococci for prevention of dental caries. Cationic liposome, nisin-loaded dipalmitoylphosphatidylcholine/phytosphingosine, exhibited higher bactericidal activities than those of electroneutral liposome and anionic liposome. Bactericidal efficiency of the cationic liposome revealed that the vesicles exhibited sustained inhibition of glucan synthesis and the lowest rate of release of nisin from the vesicles. The optimizing ability of cationic liposomeencapsulated nisin that exploit the sustained preventive features of an anti-streptococcal strategy may improve prevention of dental caries.
\end{abstract}

Keywords: Cationic liposome, Dental caries, Insoluble glucan, MIC, Nisin, Streptococcus mutans.

\section{INTRODUCTION}

Dental caries caused by Streptococcus mutans is a global oral health problem. Glucosyltransferases of the cariogenic streptococci synthesize insoluble glucan-biofilm on the tooth surfaces, and the glucan contributes to the formation of tartar and plaque [1]. The insoluble glucan on tooth surfaces mediates bacterial colonization and is an essential element in the pathogenesis of dental caries [2]. Therefore, a key for the prevention of dental caries is bactericidal ability against $S$. mutans [3]. Furthermore, inhibition of insoluble glucan synthesis is required to maintain oral health.

Nisin is an antimicrobial peptide, produced by Lactococcus lactis subsp. lactis. This class IA lantibiotic has the ability to inhibit Gram-positive bacteria [4]. Nisin has been identified to suppress S. mutans in vitro [5]. Nisin does not appear to be toxic to normal human gingival fibroblasts and epithelial cells [6]. However, nisin is negatively affected by proteolytic degradation, oxidation, and its own insolubility in vitro and in vivo. Liposomes are accepted to encapsulate several bioactive molecules, and these molecules are entrapped in the lipid layer or in the aqueous core depending on

\footnotetext{
* Address correspondence to this author at the, Department of Preventive Medicine and Public Health, National Defense Medical College, Tokorozawa 359-8513, Japan; Tel: +81-4 (2995)1563; Fax: +81-4(2996) 5195; E-mail: yamakami@ndmc.ac.jp
} 
the molecule's inherent lipophilicity or hydrophilicity [7]. Thus, liposomes have been demonstrated to be effective for encapsulating unstable bioactive molecules, and also safe for human consumption. Lipid selection is based on the vesicle stabilizing capacity, and the vesicles can express the complete biological ability of the encapsulated molecules. Liposomes have gained attention as biocompatible carriers, and the expected function is their ability to protect the entrapped molecules within the lipid vesicles [8]. The applications of liposome encapsulation of antimicrobial peptides have been developed for food chemistry [5]. Stabilized liposomes with a prolonged lifetime of encapsulated nisin have been investigated for maximal efficiency. For preventing dental caries, liposome-encapsulated nisin is an attractive approach [9]. We are exploiting the physical stability profile of liposome-encapsulated nisin in vitro and have observed that liposome-encapsulated nisin shows bactericidal activity against $S$. mutans but there are few data describing the ability of liposome-encapsulated nisin for preventing dental caries [10]. In recent studies, the effects of acyl chain length of phosphatidylcholine on nisin activity have been shown to influence the effectiveness of bactericidal activity against $S$. mutans and the inhibitory effect on insoluble glucan synthesis [11]. However, the outcome of our effort to effectively facilitate sustained nisin activity has not yet been encouraging. Efficiency of charged lipid on the liposomeencapsulated nisin has not been investigated with the intent of prevention for dental caries. The aim of our study was to design a candidate lipid formulation for long lasting bactericidal activity by the sustained release of nisin from the liposomes. Here we evaluated the liposome compositions formulated with charged lipids for optimizing their ability of bactericidal activity against $S$. mutans and their influences on the inhibition of insoluble glucan formation.

\section{MATERIALS AND METHODS}

\section{Preparation of Nisin}

Nisin was obtained from Lactococcus lactis subsp. lactis 11454 (American Type Culture Collection; Manassas, VA, USA) and was purified, as described previously [4, 10]. Briefly, pellets of cultured L. lactis were suspended in $70 \%$ isopropanol $/ 0.1 \%$ trifluoroacetic acid. The suspension was centrifuged at 7,000 $\mathrm{g}$ for $10 \mathrm{~min}$, and the supernatant fluid was evaporated. The clarified materials were subsequently subjected to reverse-phase HPLC using a C-8 column (YMC Inc., Kyoto, Japan) pre-equilibrated with $0.1 \%$ trifluoroacetic acid, and the resulting profile was monitored at $220 \mathrm{~nm}$. For elution of nisin, the column was developed in a linear gradient $70 \%$ isopropanol $/ 0.1 \%$ trifluoroacetic acid. To determine the purity of nisin, peak fractions were re-run on the C-8 column, and the purity was assessed using chromatography at $220 \mathrm{~nm}$. Nisin of $>95 \%$ purity was used in this study. The purified fractions were passed through a membrane filter with $0.22 \mu \mathrm{m}$ pore size. The solutions of $0.3 \mu \mathrm{mol}$ each of the purified nisin were placed into vial and then lyophilized. The vials were stored at $4^{\circ} \mathrm{C}$ until use.

\section{Preparation of Liposome-encapsulated Nisin}

We used the following lipids: dipalmitoylphosphatidylcholine (DPPC), dipalmitoylphosphatidylethanolamine (DPPE) and stearoylphytosphingosine (SPS) as the electroneutral lipid; dipalmitoylphosphatidylglycerol (DPPG) as the anionic lipid; and phytosphingosine (PS) as the cationic lipid (Avanti Polar Lipids. Inc., Alabaster, AL, USA). Two lipid molar formations: DPPC/DPPG, DPPC/DPPE, DPPC/SPS, and DPPC/PS (85:15 mol\%) were prepared for liposomes. DPPC (100\%) was placed as the control liposome. The liposomes were prepared using the freeze-dried empty liposomes (FDEL) method [12]. Briefly, appropriate amounts of the each composition of lipids were dissolved in chloroform, and the solvent was removed by evaporation. These lipids were evacuated and then hydrated with water at $60^{\circ} \mathrm{C}$. The dispersions were passed through a membrane filter with a $0.22 \mu \mathrm{m}$ pore size. The dispersions of $40 \mu \mathrm{mol}$ as the lipids were placed in vial, and then lyophilized. The vials were stored at $4^{\circ} \mathrm{C}$ until use. To prepare liposomeencapsulated nisin, the lyophilized nisin in a vial was dissolved with $1 \mathrm{ml}$ of $5 \mathrm{mM}$ sodium citrate (pH 5.0). The materials were transferred to the vial of FDEL $(40 \mu \mathrm{mol})$ and then vortexed, thus obtaining the compositions of 0.3 $\mu \mathrm{mol}$ nisin/40 $\mu \mathrm{mol}$ lipids as the liposome-encapsulated nisin. To determine the efficiency of encapsulation of nisin, 20 $\mu \mathrm{l}$ of liposomes were mixed with $80 \mu \mathrm{l}$ of an aqueous solution of $2 \%$ Triton $\mathrm{X}-100$ and incubated at $37^{\circ} \mathrm{C}$ to release nisin. Encapsulation efficiency was determined by comparing the amounts of starting and released nisin using a CBQCA protein assay kit (Molecular Probes, Eugene, OR, USA) according to the manufacturer's instructions.

\section{Assay of Bactericidal Activity of Liposome-encapsulated Nisin}

Determinations of the minimum inhibitory concentrations (MIC) as indicative of the bactericidal activity of liposome preparations against $S$. mutans 10449 (American Type Culture Collection) were performed using the broth dilution method [13]. Amounts of $S$. mutans were measured the turbidity at $600 \mathrm{~nm}$ [10]. The liposome preparations 
were added to 96 well flat-bottom microplates at final concentrations of 5-250 pmol in $50 \mu 1$ of $10 \mathrm{mM}$ sodium citrate (pH 7.0). S. mutans 10449 in $50 \mu$ l of Todd Hewitt Broth (THB; Invitrogen, Carlsbad, CA, USA) containing resazurin dye (Sigma-Aldrich, St. Louis, MO, USA) were added to the wells; the plates were incubated at $37^{\circ} \mathrm{C}$ for $4 \mathrm{~h}$ and then assayed colorimetrically at $570 \mathrm{~nm}$ [14]. The MIC values were defined as the lowest amounts of nisin that inhibited the growth of $S$. mutans.

\section{Assay of the Inhibitory Effect of Liposome-encapsulated Nisin on Insoluble Glucan Synthesis}

The effects of nisin-loaded liposome on the synthesis of insoluble glucan were assayed by the method of our previous report [10]. Insoluble glucan was generated using $S$. mutans cultured in 96 well flat-bottom microplates. Assays were performed using a solution containing S. mutans in $50 \mu \mathrm{l}$ of THB, $50 \mu \mathrm{l}$ of $1.0 \%$ sucrose, and bacteriocin (naked nisin, $75 \mathrm{pmol}$; and nisin-loaded liposome, $75 \mathrm{pmol}$ as the nisin equivalent) in $100 \mu \mathrm{l}$ of 5 mM sodium citrate (pH 7.0) were added to individual wells. The plates were incubated at $37^{\circ} \mathrm{C}$, and insoluble glucans were quantified at appropriate time points. Insoluble glucans in each well were washed with $10 \mathrm{mM}$ sodium phosphate buffer ( $\mathrm{pH} 7.0)$ and dissolved with $200 \mu \mathrm{l}$ of $1.0 \mathrm{M}$ sodium hydroxide. The amounts of alkaline solubilized glucan were quantified by the phenol-sulfuric acid method [15]. Inhibition of glucan synthesis was determined according to the amounts of insoluble glucan in each well compared with those in wells lacking nisin or liposomes.

\section{In Vitro Nisin Release Assay from Liposome-encapsulated Nisin}

In vitro assays of nisin release from the liposomes were performed by the method of previous report [10]. Nisinloaded liposome (100 pmol as the nisin equivalent) in $200 \mu 1$ of $5 \mathrm{mM}$ sodium citrate $(\mathrm{pH} 7.0)$ containing $0.01 \%$ Triton $\mathrm{X}-100$ was incubated in 96 -well microplates at $37^{\circ} \mathrm{C}$. At the appropriate times, the supernatants obtained by centrifuging the liposomes at $10,000 \times \mathrm{g}$ for $10 \mathrm{~min}$ were assayed fluorometrically using the CBQCA quantitation kit. The amounts of nisin are expressed as the cumulative percentages of nisin released at each time point.

\section{Statistical Analysis}

Assays were performed in triplicate, and values are reported as the mean. The standard deviation was $<5 \%$ for all assays. The statistical significance of differences between groups was assessed using the Student $t$-test, and $P<0.05$ was considered statistically significant.

\section{RESULTS}

The encapsulation efficiencies of nisin to the FDEL were between $70 \%-80 \%$, with the differences possibly due to the variation within the experiment. The first step of this study was to examine the effects of charge on liposomeencapsulated nisin as the efficiency for bactericidal activity against $S$. mutans in vitro. To assess the contribution of charge on vesicles, we examined the effect of the cationic lipid PS, the anionic lipid DPPG, and the electroneutral lipids DPPE and SPS at pH 7.0. The results of the MIC assay, presented in Fig. (1) indicated that the cationic liposome, nisinloaded DPPC/PS, showed 2 fold higher bactericidal activity against $S$. mutans than DPPC-encapsulated nisin as the control liposome $(P<0.05)$, and the liposome achieved most effective bactericidal activity $(75$ pmol as the nisin equivalent). The control liposome nisin-loaded DPPC exhibited slightly higher MIC value (150 pmol as the nisin equivalent) than that of naked nisin $(175 \mathrm{pmol})$ in our assay conditions. The neutral liposomes nisin-loaded DPPC/DPPE and DPPC/SPS showed 1.3 and 1.7 fold higher MIC values, respectively (112 and 87.5 pmol as the nisin equivalent, respectively) than the control liposome. In contrast, the anionic liposome nisin-loaded DPPC/DPPG showed the lowest bactericidal effect ( $250 \mathrm{pmol}$ as the nisin equivalent) in liposomes, and the effect was lower (0.6 fold) than that of the control liposome. Thus, the nisin-loaded liposome vesicles with positively charged lipids PS apparently increased the bactericidal activity.

The next step of this study was to examine the inhibition of insoluble glucan synthesis by liposome preparations with the above lipid compositions. The findings shown in Fig. (2) indicated the marked inhibition of insoluble glucan synthesis by the cationic liposome nisin-loaded DPPC/PS with 3.4 fold higher inhibition than that of DPPCencapsulated nisin as the control at the endpoint of incubation $(3.75 \mathrm{~h} ; P<0.05)$. DPPC-encapsulated nisin at 75 pmol as the nisin equivalent exhibited higher inhibition ( 1.4 fold) to insoluble glucan synthesis compared with the effect of naked nisin. The neutral liposomes nisin-loaded DPPC/DPPE and DPPC/SPS achieved significantly higher inhibition of glucan synthesis than that of DPPC-encapsulated nisin (1.4 and 1.7 fold, respectively). In contrast, the anionic liposomes nisin-loaded DPPC/DPPG achieved an inhibition equivalent to that of naked nisin, and the inhibiting profile 
was lower than that of the buffer alone ( 0.67 fold). The anionic liposome-encapsulated nisin demonstrated the lowest inhibitory effect of insoluble glucan synthesis (at $3.75 \mathrm{~h}$ ). Inhibition of the glucan synthesis by these charged liposomeencapsulated nisin was essentially equivalent up to $1.5 \mathrm{~h}$ incubation, and the composition of nisin-loaded DPPC/PS was apparently increasing the inhibition after $2.25 \mathrm{~h}$ incubation. To evaluate the potential significance of bactericidal activity and inhibitory effect of charged vesicles on glucan synthesis, we examined the in vitro release assay of these encapsulated nisin (Fig. 3). The cationic liposome nisin-loaded DPPC/PS achieved 41\% of releasing capacity based upon the $2 \mathrm{~h}$ incubation. At the same time point, DPPC-encapsulated nisin as the control liposome released $80 \%$ of nisin $(P<0.05)$. The anionic liposome nisin-loaded DPPC/DPPG released nisin at similar levels of DPPC-encapsulated nisin as the control. Thus, the in vitro release assay of the liposome formulations showed that the extent of cationic vesicle is significant.

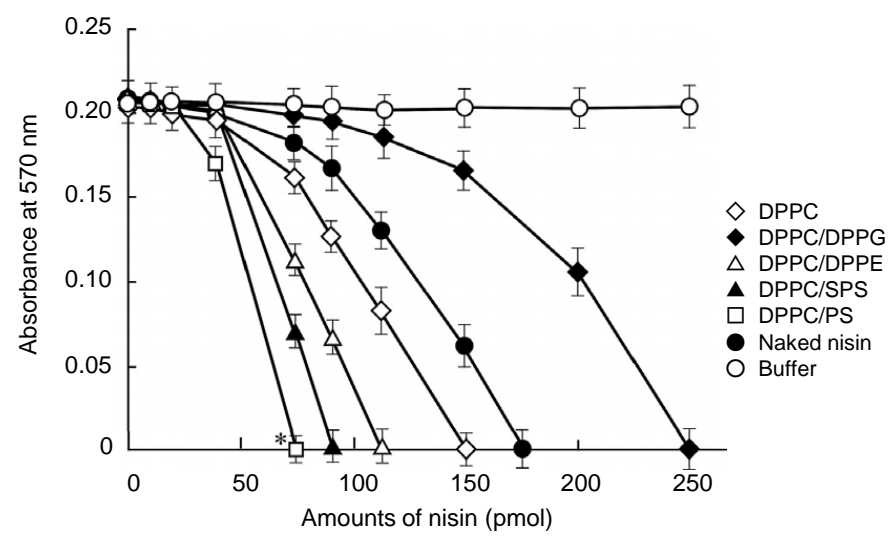

Fig. (1). Bactericidal activity of liposome-encapsulated nisin against Streptococcus mutans. Exponentially proliferating cultures of $S$. mutans 10449 (turbidity of 0.02 at $600 \mathrm{~nm}$ ) were incubated for $4 \mathrm{~h}$ at $37^{\circ} \mathrm{C}$ in $0.2 \mathrm{ml}$ of Todd Hewitt Broth in the presence of nisinloaded liposome, an equivalent concentration of naked nisin, or buffer. A minimum inhibitory concentration (MIC) assay as indicative of the bactericidal activity of individual samples was quantified using resazurin dye. Symbols indicate the lipid formulations of liposomes and controls. $* P<0.05$ indicates a significant difference of MIC values of the reaction mixtures containing DPPC/PS-encapsulated nisin compared with those of the control liposome, nisin-loaded DPPC. Values represent the mean of triplicate replications and the bars represent the standard deviation of sample means.

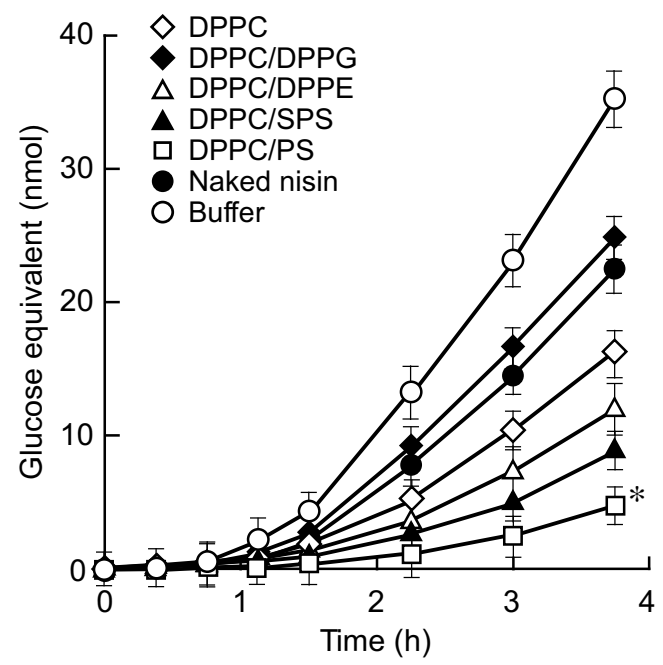

Fig. (2). Effect of liposome-encapsulated nisin on insoluble glucan synthesis by Streptococcus mutans. Exponentially proliferating cultures of $S$. mutans 10449 (turbidity of 0.02 at $600 \mathrm{~nm}$ ) were incubated in Todd Hewitt Broth containing $0.25 \%$ sucrose at $37^{\circ} \mathrm{C}$ for $3.75 \mathrm{~h}$ in the presence of liposome-encapsulated nisin at concentrations equivalent to that of naked nisin, or naked nisin alone. Symbols indicate the lipid formulations of liposomes and controls. $* P<0.05$ compared with the control liposome nisin-loaded DPPC. Values represent the mean of triplicate replications and the bars represent the standard deviation of sample means. 


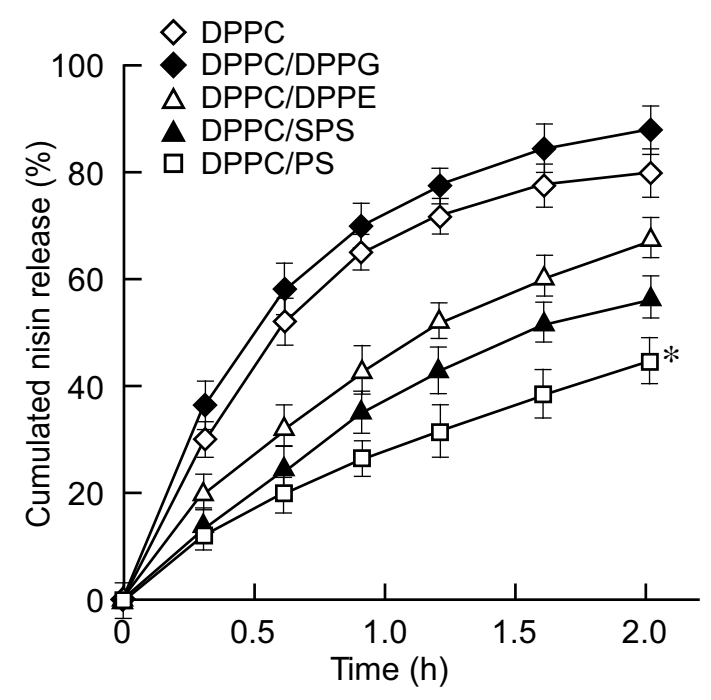

Fig. (3). In vitro nisin release assay from liposome-encapsulated nisin. Liposome-encapsulated nisin was incubated in $10 \mathrm{mM}$ sodium citrate ( $\mathrm{pH} 7.0$ ) containing $0.01 \%$ Triton $\mathrm{X}-100$ at $37^{\circ} \mathrm{C}$. At the appropriate incubation periods, amounts of nisin released into the supernatant at $10,000 \times \mathrm{g}$ for $10 \mathrm{~min}$ were quantitated. The levels of nisin are expressed as the cumulative percentage release. The maximum value was defined as $100 \%$ release. Symbols indicate the lipid formulations of liposomes. The assays were performed in triplicates at each time point, and the bars represent the standard deviation of sample means. ${ }^{*} P<0.05$ compared with the group of DPPC-encapsulated nisin.

\section{DISCUSSION}

We are taking steps toward optimizing liposome-based nisin to improve the efficiency of bactericidal activity against cariogenic streptococci for the purpose of prevention of dental caries [10]. Our recent study indicated that the acyl chain length of lipids in liposome affects the retaining of encapsulated nisin and suggests that the hydrophobicity of vesicle is a factor necessary for the sustainable bactericidal activity of nisin [11]. Furthermore, we focused the investigation regarding the effects of charge of composed lipids on liposome-encapsulated nisin for the bactericidal activity against $S$. mutans, and inhibition of insoluble glucan synthesis by the streptococci for the purpose of optimizing the sustained effect of the encapsulated nisin. For example, there are reports that the charge on vesicles affects the effective induction of bactericidal activity of the liposome-encapsulated antibiotics [16]. Some reports have determined that positively charged liposomes evoke more effective bioactivity of encapsulated molecules than nonionic vesicles $[17,18]$. These reports determined that charged lipids interact with the target molecules. We observed that nisin-loaded DPPC/PS as the cationic liposome was more effective in terms of bactericidal activity than electroneutral or anionic liposome-encapsulated nisin at $\mathrm{pH} 7.0$ in our experimental conditions. A related report has shown that the level of release of encapsulated peptides at the preferred ratios from cationic liposomes was sustained in comparison with that of neutral or anionic liposomes [19]. Furthermore, it is possible that cationic lipids such as PS interact with membrane of streptococci, but anionic or neutral lipids do not associate with the membrane. In this study, combinations of naked nisin plus appropriate empty liposomes or empty liposomes alone served as the control for individual liposome preparations. However, the combination of empty cationic liposome plus naked nisin or empty cationic liposome alone did not affect the evoked bactericidal activity against $S$. mutans as compared with cationic liposome-encapsulated nisin. Because cationic liposomes are effective carriers for several molecules, properties of the vesicles indicate that the vesicles not only maintain the encapsulated nisin at a stable state but also exert bactericidal activity against $S$. mutans by sustained releasing the nisin [17]. In addition, cationic liposomes show low toxicity to human cells and may have biomedical applications [19]. Thus, we expect to exploit cationic liposome-encapsulated nisin as the prophylactics to dental caries. Chemical features of liposome vesicles are being investigated to determine their use for encapsulating the bioactive molecule nisin in vitro. Additional efforts aimed at optimizing the vesicle formulation are expected, and our continuing studies should improve the oral health.

\section{CONCLUSION}

In this study, the cationic liposome nisin-loaded DPPC/PS could exert a sustained bactericidal effect against $S$. mutans and its inhibition of insoluble glucan synthesis in vitro. Optimizing the ability of cationic liposome-encapsulated nisin may improve oral health. 


\section{CONFLICT OF INTEREST}

The authors confirm that this article content has no conflict of interest.

\section{ACKNOWLEDGEMENTS}

Declared none.

\section{REFERENCES}

[1] Paes Leme AF, Koo H, Bellato CM, Bedi G, Cury JA. The role of sucrose in cariogenic dental biofilm formation: new insight. J Dent Res 2006; 85(10): 878-87. [http://dx.doi.org/10.1177/154405910608501002] [PMID: 16998125]

[2] Germano F, Bramanti E, Arcuri C, Cecchetti F, Cicciù M. Atomic force microscopy of bacteria from periodontal subgingival biofilm: Preliminary study results. Eur J Dent 2013; 7(2): 152-8. [http://dx.doi.org/10.4103/1305-7456.110155] [PMID: 24883019]

[3] Cury JA, Rebelo MA, Del Bel Cury AA, Derbyshire MT, Tabchoury CP. Biochemical composition and cariogenicity of dental plaque formed in the presence of sucrose or glucose and fructose. Caries Res 2000; 34(6): 491-7. [http://dx.doi.org/10.1159/000016629] [PMID: 11093024]

[4] Field D, Connor PM, Cotter PD, Hill C, Ross RP. The generation of nisin variants with enhanced activity against specific gram-positive pathogens. Mol Microbiol 2008; 69(1): 218-30. [http://dx.doi.org/10.1111/j.1365-2958.2008.06279.x] [PMID: 18485077]

[5] Prombutara P, Kulwatthanasal Y, Supaka N, Sramala I, Chareonpornwattana S. Production of nisin-loaded solid lipid nanoparticles for sustained antimicrobial activity. Food Contr 2012; 24: 184-90. [http://dx.doi.org/10.1016/j.foodcont.2011.09.025]

[6] Akerey B, Le-Lay C, Fliss I, Subirade M, Rouabhia M. In vitro efficacy of nisin Z against Candida albicans adhesion and transition following contact with normal human gingival cells. J Appl Microbiol 2009; 107(4): 1298-307. [http://dx.doi.org/10.1111/j.1365-2672.2009.04312.x] [PMID: 19486401]

[7] Matsui M, Shimizu Y, Kodera Y, Kondo E, Ikehara Y, Nakanishi H. Targeted delivery of oligomannose-coated liposome to the omental micrometastasis by peritoneal macrophages from patients with gastric cancer. Cancer Sci 2010; 101(7): 1670-7. [http://dx.doi.org/10.1111/j.1349-7006.2010.01587.x] [PMID: 20507320]

[8] Ikegami S, Yamakami K, Ono T, et al. Targeting gene therapy for prostate cancer cells by liposomes complexed with anti-prostate-specific membrane antigen monoclonal antibody. Hum Gene Ther 2006; 17(10): 997-1005. [http://dx.doi.org/10.1089/hum.2006.17.997] [PMID: 17032155]

[9] Tong Z, Dong L, Zhou L, Tao R, Ni L. Nisin inhibits dental caries-associated microorganism in vitro. Peptides 2010; 31(11): 2003-8. [http://dx.doi.org/10.1016/j.peptides.2010.07.016] [PMID: 20688123]

[10] Yamakami K, Tsumori H, Sakurai Y, Shimizu Y, Nagatoshi K, Sonomoto K. Sustainable inhibition efficacy of liposome-encapsulated nisin on insoluble glucan-bilfilm synthesis. Pharm Biol 2013; 51: 267-70. [http://dx.doi.org/10.3109/13880209.2012.717227] [PMID: 23116173]

[11] Yamakami K, Tsumori H, Shimizu Y, Sakurai Y, Nagatoshi K, Sonomoto K. Effect of liposomal phosphatidylcholine acyl chain length on the bactericidal activity of liposome-encapsulated nisin on cariogenic Streptococcus mutans. J Dent Health Oral Disord Ther 2014; 1: 17-20.

[12] Kikuchi H, Suzuki N, Ebihara K, et al. Gene delivery using liposome technology. J Control Release 1999; 62(1-2): 269-77. [http://dx.doi.org/10.1016/S0168-3659(99)00047-4] [PMID: 10518660]

[13] Kuwano K, Tanaka N, Shimizu T, Nagatoshi K, Nou S, Sonomoto K. Dual antibacterial mechanisms of nisin Z against Gram-positive and Gram-negative bacteria. Int J Antimicrob Agents 2005; 26(5): 396-402. [http://dx.doi.org/10.1016/j.ijantimicag.2005.08.010] [PMID: 16226432]

[14] Sakuma Y, Washio J, Sasaki K, Takahashi N. A high-sensitive and non-radioisotopic fluorescence dye method for evaluating bacterial adhesion to denture materials. Dent Mater J 2013; 32(4): 585-91. [http://dx.doi.org/10.4012/dmj.2013-060] [PMID: 23903640]

[15] Tsumori H, Shimamura A, Sakurai Y, Yamakami K. Inhibitory effects of mutanase and dextranase on the formation of insoluble glucanbiofilms by cariogenic streptococci. Food Funct 2012; 10: 37-42.

[16] Zhao W, Zhuang S, Qi XR. Comparative study of the in vitro and in vivo characteristics of cationic and neutral liposomes. Int J Nanomedicine 2011; 6: 3087-98.

[PMID: 22163162]

[17] Krasnici S, Werner A, Eichhorn ME, et al. Effect of the surface charge of liposomes on their uptake by angiogenic tumor vessels. Int J Cancer 2003; 105(4): 561-7.

[http://dx.doi.org/10.1002/ijc.11108] [PMID: 12712451]

[18] Villasmil-Sánchez S, Drhimeur W, Ospino SC, Rabasco Alvarez AM, González-Rodríguez ML. Positively and negatively charged liposomes as carriers for transdermal delivery of sumatriptan: in vitro characterization. Drug Dev Ind Pharm 2010; 36(6): 666-75. 
[http://dx.doi.org/10.3109/03639040903419640] [PMID: 20136486]

[19] Carmona-Ribeiro AM, de Melo Carrasco LD. Cationic antimicrobial polymers and their assemblies. Int J Mol Sci 2013; 14(5): 9906-46. [http://dx.doi.org/10.3390/ijms14059906] [PMID: 23665898]

(c) Yamakami et al; Licensee Bentham Open.

This is an open access article licensed under the terms of the Creative Commons Attribution-Non-Commercial 4.0 International Public License (CC BY-NC 4.0) (https://creativecommons.org/licenses/by-nc/4.0/legalcode), which permits unrestricted, non-commercial use, distribution and reproduction in any medium, provided the work is properly cited. 